\title{
Processos Cognitivos Visuoespaciais e Ambiente Visual Circundante: Implicações Educacionais
}

\author{
Pedro F. S. Rodrigues ${ }^{1}$ \\ Universidade de Aveiro
}

\begin{abstract}
RESUMO - Nesta revisão de literatura, abordamos a importância da atenção seletiva, da inibição e da memória de trabalho na aprendizagem das crianças. Começamos por apresentar as suas definições e principais mecanismos funcionais. Apresentamos igualmente conclusões de vários estudos que abordam a importância desses processos, sobretudo em tarefas visuoespaciais. Finalmente, abordamos o papel que o ambiente visual circundante desempenha na aprendizagem, chamando a atenção para uma lacuna que se verifica em grande parte dos estudos: a sua pouca validade ecológica. É também defendido que o ambiente visual externo deve ser considerado nos modelos explicativos dos processos cognitivos básicos. Conclui-se o trabalho alertando para a necessidade de se estudar de forma mais sistemática a relação entre estes dois elementos (cognição e ambiente).
\end{abstract}

Palavras-chave: atenção seletiva, inibição, memória de trabalho, aprendizagem, ambiente visual circundante, validade ecológica

\section{Visuospatial Cognitive Processes and Visual Surrounding Environment: Educational Implications}

\begin{abstract}
This literature review focuses on the importance of selective attention, inhibition and working memory in children's learning. We start by presenting the definition and functional mechanisms of these cognitive processes. We then present the main results of several studies that report the importance of these processes, particularly in visuospatial tasks. We also discuss the role of the visual surrounding environment in learning, particularly with respect to an existing gap in most studies: Their low ecological validity. We argue for the importance of considering the visual external environment when trying to explain performance in basic cognitive processes. Finally, we stress the need for more empirical studies exploring in a systematic way the relation between these two elements (cognition and environment).
\end{abstract}

Keywords: selective attention, inhibition, working memory, learning, visual surrounding environment, ecological validity

Compreender os processos cognitivos e a forma como decorre o seu desenvolvimento é um aspecto que tem preocupado a comunidade científica ao longo dos anos, particularmente quando falamos em crianças em idade escolar. O conhecimento dos processos atencionais e mnemônicos, assim como a sua relação com a aprendizagem é um aspecto crucial para a eficácia dos vários profissionais que trabalham com essa faixa etária, nomeadamente os psicólogos e professores. Este trabalho apresenta uma revisão de literatura que aborda a importância da atenção seletiva, da inibição e da memória de trabalho na aprendizagem das crianças em idade escolar. A abordagem aqui apresentada refere-se sobretudo ao componente visuoespacial dos processos mencionados e focaliza a apresentação dos principais resultados e conclusões de várias investigações. O trabalho também aponta possíveis linhas de investigação futuras, dando particular enfoque a uma lacuna que a maioria dos estudos apresenta: a sua pouca validade ecológica. Comecemos por apresentar uma definição de validade ecológica: diz respeito aos métodos científicos com que estudamos comportamentos humanos

1 Endereço para correspondência: Departamento de Educação e Psicologia, Universidade de Aveiro, Campus Universitário de Santiago, Aveiro, Portugal. CEP. 3810-193.E-mail: pedro.filipe@ua.pt em contextos ambientais específicos, o mais próximos possível dos contextos reais em que esses ocorrem (Davids, 1988). A validade ecológica relaciona-se em grande medida com o ambiente circundante ao indivíduo, que tem sido considerado em contexto educacional (Fisher, Godwin, \& Seltman, 2014), assim como no campo da Psicologia Cognitiva Básica (Rodrigues \& Pandeirada, 2015), embora a sua evidência empírica seja ainda muito escassa, tal como se pode compreender ao longo do presente artigo.

$\mathrm{O}$ trabalho dá particular destaque à influência do ambiente visual circundante na aprendizagem, terminando com uma proposta de procedimento de investigação em meio educacional. Essa proposta fundamenta-se na interação indivíduo-ambiente circundante. Esta defende que não se deve investigar o indivíduo e consequentemente o seu comportamento sem se considerar também a influência que o ambiente circundante nele exerce (Barrett, Davies, Zhang, \& Barrett, 2015; Gifford, 2007). Aumentar a validade ecológica dos procedimentos experimentais torna-se, por isso, vital para se compreender com maior exatidão os processos cognitivos que baseiam as atividades do cotidiano, de que é exemplo a aprendizagem escolar das crianças. 


\section{Atenção Seletiva, Inibição, Memória de Trabalho e Aprendizagem: Definições}

Apresentar qualquer um dos conceitos não é uma tarefa simples e carece até de consensualidade. Contudo, as definições que se apresentam seguidamente procuram ser uma convergência de vários autores, que ao longo das últimas décadas se têm dedicado ao estudo dos referidos processos cognitivos.

Comecemos por definir atenção. Trata-se de um processo complexo que nos permite focar os recursos cognitivos em uma dada informação ambiental, podendo dirigir-se a uma só tarefa ou ser distribuída por várias (Lachman, Lachman, \& Butterfield, 2015; Posner, 2011). É um processo cognitivo essencial para diversos outros, nomeadamente para a percepção, a ação, a memória e a aprendizagem, sendo, por isso, considerado a primeira etapa dos sistemas de pensamento e de ação (Amso \& Scerif, 2015). Existem diversos tipos de atenção de que são exemplo: atenção seletiva, dividida ou sustentada (Styles, 2005). Neste trabalho dá-se particular enfoque ao processo de atenção seletiva, que diz respeito à capacidade humana de se direcionarem os recursos cognitivos para informações relevantes a determinados objetivos, enquanto se ignoram as irrelevantes (Gazzaley \& Nobre, 2012; Posner, 2011; Styles, 2005). No caso concreto do presente trabalho, focamo-nos na informação visuoespacial. Diariamente, na relação que estabelecemos com o meio ambiente, estamos expostos a uma grande variedade e quantidade de estímulos, nomeadamente do foro visuoespacial, não sendo possível processá-los todos em simultâneo (Amso \& Scerif, 2015). O que explica em grande parte a seleção que fazemos constantemente desses estímulos visuoespaciais é o controle dicotômico atencional efetuado quer pelo processamento bottom-up ou ascendente, quer pelo processamento topdown ou descendente. O primeiro permite-nos processar os estímulos externos de acordo com a sua relevância e caraterísticas próprias (e.g., prestar atenção a um estímulo visual colorido). A rede neuronal do processamento bottomup designa-se por rede atencional ventral e é constituída pela junção temporoparietal, bem como pelo córtex frontal ventral (Katsuki \& Constantinidis, 2012; Petersen \& Posner, 2012). Por outro lado, o processamento top-down, baseado nas nossas influências internas, permite-nos focar a atenção nos estímulos que efetivamente são relevantes para uma dada tarefa, enquanto ignoramos outros que são irrelevantes (Zanto, Hennigan, Östberg, Clapp, \& Gazzaley, 2010). A rede neuronal do processamento top-down é a rede atencional dorsal, constituída nomeadamente pelos campos oculares frontais e pelo sulco intraparietal/lobo parietal superior (Petersen \& Posner, 2012).

Outro processo cognitivo igualmente importante no nosso dia-a-dia e que se encontra estreitamente relacionado com a atenção seletiva é a inibição. Esta, também designada por controle inibitório, diz respeito à capacidade de os indivíduos inibirem respostas a estímulos irrelevantes ou distratores a um dado objetivo, que habitualmente interrompem o curso de uma ação ou resposta (Barkley, 1997, 2001; Diamond, Barnett, Thomas, \& Munro, 2007). O controle inibitório parece ser, assim, um processo cognitivo complementar ao de atenção seletiva, estando os dois interligados na execução de variadas tarefas do nosso dia-a-dia (Booth et al., 2003; Terry, 1995). O objetivo fisiológico do controle inibitório é a supressão de entradas internas (por intermédio, por exemplo, da memória) e externas (por intermédio dos sentidos) que possam interferir no comportamento em curso. Dificuldades no controle inibitório estão comumente associadas a impulsividade. Um dos indicadores do desenvolvimento psicossocial da criança é precisamente o desenvolvimento progressivo do controle inibitório sobre os estímulos internos e externos. De fato, à medida que a criança cresce, verificase o desenvolvimento dos seus processos atencionais, o que a leva a tornar-se mais capaz de focalizar e de centrar a sua atenção nas tarefas em curso, tornando-a menos distraída, menos impulsiva e, portanto, mais capaz de exercer o autocontrole (Fuster, 2002; Kirkham, Cruess, \& Diamond, 2003; Zelazo, Craik, \& Booth, 2004).

Já a memória diz respeito ao processo cognitivo por via do qual codificamos, armazenamos e recuperamos as informações, designadamente as presentes no meio ambiente (Radvansky, 2011). Para melhor percebermos o seu funcionamento, façamos uma analogia a um computador. Imaginemos que queremos registar uma determinada informação em um documento digital, gravando esse documento no disco do computador. Nessa analogia, a codificação mnésica diria respeito ao processo de escrita da informação através do teclado do computador, enquanto o armazenamento diria respeito ao processo de gravação do documento no disco do computador. A recuperação seria análoga à procura e abertura do documento digital a partir do disco do computador quando estamos a realizar uma tarefa e necessitamos de consultar o documento para a sua execução (Gleitman, Fridlund, \& Reisberg, 2007; Styles, 2005). Existem vários tipos e modelos que tentam explicar os processos de memória, contudo um dos mais estudados e ao qual se dá particular destaque neste artigo diz respeito ao modelo de memória de trabalho (Baddeley, 1992, 2010, 2012). Esta refere-se ao sistema de armazenamento e manipulação temporária da informação que atua durante a realização de um conjunto de outras tarefas cognitivas, tais como na compreensão, na aprendizagem e no raciocínio (Baddeley, 2010). Consiste na capacidade mnésica de manter e/ou manipular, por curtos períodos de tempo, as informações relevantes a uma dada tarefa, tratando-se, por isso, de um sistema ativo de armazenamento da informação (Baddeley, 1992, 2010). Segundo recentes atualizações do referido modelo (Baddeley, 2012), esse tipo de memória compreende quatro componentes: o executivo central, a alça fonológica, o esboço visuoespacial, bem como o buffer episódico. $\mathrm{O}$ primeiro engloba capacidades de atenção e seleção de informação, que permitem selecionar as informações a uma dada tarefa, ignorando os estímulos irrelevantes/distrativos. O executivo central permite ainda a ligação entre informação que está a ser processada em um determinado momento e aquela que está armazenada na memória a longo-prazo. Esse componente justifica que a atenção seletiva e a memória de trabalho apresentem uma estreita relação bidirecional, influenciando-se mutuamente (de Fockert, Rees, Frith, \& Lavie, 2001; Rutman, Clapp, Chadick, \& Gazzaley, 2010). A alça fonológica é responsável pelo processamento do 
material verbal, ou seja, é fundamental para a coerência e compreensão dos discursos. Trata-se de uma componente da memória de trabalho essencial para a aquisição de nova informação verbal, nomeadamente a aquisição de palavras. O esboço visuoespacial é responsável pela manipulação e armazenamento de informação visual e espacial, tendo um papel basilar na orientação espacial e no conhecimento geográfico. Além disso, o esboço visuoespacial é ainda indispensável a tarefas acadêmicas, nomeadamente na leitura, uma vez que, entre outras funções, permite a representação da página, bem como a movimentação dos olhos com a precisão necessária para uma leitura coerente. Já o buffer episódico é responsável pela integração das informações verbal e espacial, bem como destas com a memória a longo-prazo, integrando informações provenientes do exterior (ambiente circundante) ou do interior (memória a longo-prazo; Baddeley, 2010, 2012). O buffer episódico tem um papel fundamental na evocação das memórias a longoprazo, já que durante esse processo, os traços de memória são nele reunidos, para, em seguida, serem organizados e editados, surgindo finalmente como um material "lembrado" (Baddeley, 1992, 2010). Todo esse processo é mediado, entre outros, pelo córtex pré-frontal, estrutura neocortical que envia e recebe projeções de praticamente todos os sistemas corticais sensoriais, sistemas motores, assim como de muitas estruturas subcorticais, e essencial aos processos cognitivos responsáveis pelo planeamento e execução das ações (Blair \& Razza, 2007; Jurado \& Rosselli, 2007; Miller \& Cohen, 2001). Uma das caraterísticas mais consensuais acerca da memória de trabalho diz respeito ao fato de se tratar de um sistema de memória rápido, durando apenas alguns segundos, com capacidade limitada o suficiente para memorizarmos um número de telefone e o esquecermos logo em seguida a efetuarmos um telefonema, por exemplo (Baddeley, 2010). A memória de trabalho permite a realização de outros processos cognitivos, como raciocínio ou aprendizagem, estando na base de tarefas como leitura, compreensão ou operações aritméticas. Com esses exemplos, facilmente percebemos a importância desse tipo de memória no processo de aprendizagem, que exige uma constante manipulação da informação (e.g., memória de trabalho e leitura: Arrington, Kulesz, Francis, Fletcher, \& Barnes, 2014; memória de trabalho e matemática: Friso-van den Bos, van der Ven, Kroesbergen, \& van Luit, 2013).

Uma das principais funções do sistema educativo é a formação dos alunos, que implica aquisição, compreensão e posterior aplicação dos conhecimentos adquiridos em diversas circunstâncias da vida. O processo de aprendizagem está, desse modo, estreitamente interdependente de processos cognitivos como atenção, controle inibitório e memória de trabalho (Baddeley, 2010; Borella, Carretti, \& De Beni, 2008; Pinto, 2001). Antes de avançarmos no presente trabalho, parece importante apresentar-se uma definição de aprendizagem. Esta diz respeito à mudança relativamente estável no comportamento produzida essencialmente pela experiência, i.e., diz respeito ao processo pelo qual os nossos conhecimentos são adquiridos e/ou modificados. A principal caraterística da aprendizagem é a aquisição de informação (Tavares, Pereira, Gomes, Monteiro, \& Gomes, 2007). Se, em animais, essa aquisição está sobretudo relacionada com a intensidade dos estímulos; em humanos, está também relacionada com estados emocionais, bem como com a motivação (Boekaerts, 1996; Nelson \& Low, 2003). Nos humanos, o conceito de aprendizagem está intimamente relacionado com a educação e o desenvolvimento pessoal (Gleitman et al., 2007). Esse processo de aquisição de conhecimentos tem início na estimulação sensorial e termina com o seu armazenamento e recuperação (memória), através da representação interna que criamos das informações recebidas. Essa representação é influenciada por múltiplos fatores, entre eles a atenção, a repetição e a prática, bem como as crenças (Nelson \& Low, 2003; Pinto, 2001). Segundo vários dados da neurociência experimental, a aprendizagem ocorre devido à plasticidade das redes neurais que se auto-organizam em função dos estímulos externos, o que parece justificar em grande parte o processo de evolução das espécies, que ocorreu em função da pressão exercida pelo meio ambiente (Feldman, 2009; Gallistel, 2000). O processo de aprendizagem humano, sobretudo quando falamos no sistema educativo, pressupõe que, para uma criança aprender, é necessário que se desencadeie uma gestão contínua do material já memorizado, que se irá moldar e fundir aos novos conceitos adquiridos (Garner, 2009). Para esse processo ocorrer, as seguintes fases devem encontrar-se em coordenação e bom funcionamento: aquisição, retenção e evocação da informação (Garner, 2009; Pinto, 2001; Vuontela et al., 2013). Qualquer deficit em uma dessas fases parece colocar em risco o processo de aprendizagem (Gathercole et al., 2008; Gathercole, Alloway, Willis, \& Adams, 2006; Mammarella \& Cornoldi, 2005; St Clair-Thompson \& Gathercole, 2006). As funções cognitivas executivas, nomeadamente a atenção e a memória de trabalho, são essenciais para as crianças lidarem com as elevadas exigências escolares de hoje (Aronen, Vuontela, Steenari, Salmi, \& Carlson, 2005). Foquemo-nos na relação que os processos visuoespaciais de atenção, inibição e memória de trabalho têm com a aprendizagem e, por conseguinte, com o sistema educativo. Para o efeito, apresentamos, em seguida, resultados de alguns estudos.

\section{O Papel da Atenção Seletiva, da Inibição e da Memória de Trabalho na Aprendizagem}

Um dos estudos que se destaca neste artigo é o de Vuontela et al. (2013), que teve dois objetivos centrais. $\mathrm{O}$ primeiro passou por investigar o desenvolvimento dos diferentes processos cognitivos envolvidos em uma tarefa de memória de trabalho, em uma tarefa de atenção e em uma tarefa de inibição. O segundo objetivo consistiu em verificar se as funções executivas estão associadas ao funcionamento adaptativo, ao desempenho acadêmico, bem como aos sintomas emocionais e comportamentais das crianças. A atenção foi investigada através do Continuous Performance Test; a memória de trabalho visuoespacial, através de uma tarefa $n$-back (com 3 níveis: 0, 1 e 2-back); e a resposta inibitória, através de uma tarefa $\mathrm{Go} / \mathrm{No}$ Go. Utilizaram-se ainda instrumentos de avaliação do funcionamento adaptativo, do desempenho acadêmico, bem como da sintomatologia psiquiátrica. Os participantes 
foram 54 crianças divididas em três grupos: um deles foi formado por 17 crianças com idades entre os 8 e os 9 anos; o segundo, por 21 crianças com 10 anos; e o terceiro, por 16 crianças com 11 e 12 anos. Os resultados indicaram que a idade tem um papel significativo nos fatores estudados. A eficiência e fluência cognitiva aumentam com a idade, i.e., o processamento cognitivo das crianças mais novas é mais lento e menos eficiente do que o dos mais velhos, resultado igualmente verificado no desempenho da memória de trabalho, atenção e inibição. Também se verificou que as crianças mais novas apresentam menor controle inibitório (maior impulsividade) e menores escores nas avaliações do funcionamento adaptativo. $\mathrm{O}$ estudo sugeriu igualmente que o controle inibitório é a capacidade cognitiva que mais está associada a um melhor funcionamento adaptativo das crianças (aprendizagem, capacidade de trabalho e bom comportamento) e aos resultados acadêmicos, além de se encontrar associada a menos sintomas psiquiátricos. Com esse estudo, conseguimos perceber o desenvolvimento cognitivo, mesmo em crianças com poucos anos de diferença, e verificamos que os processos de atenção, controle inibitório e memória de trabalho estão associados ao funcionamento adaptativo e à aprendizagem das crianças.

Também St Clair-Thompson e Gathercole (2006) investigaram a relação entre funções executivas e o desempenho escolar. Para isso, 51 crianças $(M=11.9$ anos, $D P=0.3$ ) foram submetidas a algumas tarefas cognitivas, nomeadamente tarefas de inibição e de memória de trabalho. Para exemplificar as tarefas de inibição administradas, passamos a abordar a Stop Signal Task. Nessa tarefa, foram apresentados aos participantes dois blocos de ensaios. No primeiro, no centro da tela de um computador, foram exibidas palavras monossilábicas de um conjunto de 24; cada palavra aparecia durante $1000 \mathrm{~ms}$. As crianças foram instruídas a categorizar verbalmente cada palavra como animal ou não-animal. No segundo bloco, o procedimento adotado foi o mesmo, com a exceção de que os participantes foram instruídos a inibirem a resposta de categorização perante um determinado sinal. No que concerne à memória de trabalho, passamos a abordar a tarefa de span espacial utilizada. Nela, foram apresentadas imagens abstratas, em que cada criança categorizava como imagem em posição normal ou imagem em posição de espelho. Para além das várias tarefas, das quais só demos alguns exemplos, foram ainda recolhidas informações acerca das avaliações escolares das crianças, especificamente sobre as disciplinas de inglês, matemática e ciências. Os resultados mostraram que o desempenho da memória de trabalho esteve estreitamente relacionado com os resultados acadêmicos de inglês e de matemática, enquanto que a inibição esteve associada a bons desempenhos nas três disciplinas abordadas.

Uma das tarefas comumente utilizadas nesta área é o paradigma $n$-back, cujas tarefas exigem um trabalho contínuo de processamento da memória, que permite a comparação do efeito de diferentes níveis mnemônicos (Carlson et al., 1998). Um dos seus exemplos já foi fornecido neste trabalho, ao qual se junta o próximo estudo que passamos a expor. Aronen et al. (2005) investigaram uma amostra constituída por cerca de 60 crianças com idades compreendidas entre os 6 e os 13 anos. O estudo teve como principal objetivo avaliar como é que o desempenho em tarefas de memória de trabalho audioespacial e visuoespacial está relacionado com o desempenho acadêmico e com sintomas comportamentais e emocionais, em crianças saudáveis. A avaliação da sintomatologia comportamental e emocional foi efetuada através dos seguintes instrumentos: Child Behavior Checklist, Teacher Report Form e o Children's Depression Inventory. A memória de trabalho foi avaliada por tarefas $n$-back audioespacial e visuoespacial (0-, 1-, e 2-back). Os resultados indicaram que as crianças com baixo desempenho da memória de trabalho, tal como avaliado pelas tarefas, especialmente no que toca à memória audioespacial, apresentaram mais dificuldades atencionais, comportamentais e acadêmicas do que as crianças com bom desempenho da memória de trabalho. Um aumento do número de falsos alarmes e de omissões nas tarefas auditivas e visuais esteve associado a problemas atencionais e comportamentais. Em tarefas visuais, o número de falsos alarmes e de omissões esteve também associado a ansiedade e a sintomas depressivos. Os resultados sugerem, portanto, que os deficit de memória de trabalho podem estar na base de algumas dificuldades de aprendizagem, bem como de problemas comportamentais, nomeadamente impulsividade, dificuldades de concentração e hiperatividade. Importa ainda referir que habitualmente essas associações entre memória de trabalho e desempenho escolar são encontradas mesmo depois de controladas variáveis como o quociente de inteligência (Bull, Johnston, \& Roy, 1999; Mammarella et al., 2006).

Um dos procedimentos mais usuais em investigações que abordam o papel da memória de trabalho na aprendizagem engloba o estudo de crianças com dificuldades de aprendizagem (de Jong, 1998; Gathercole et al., 2006; Mammarella \& Cornoldi, 2005; Swanson \& Sachse-Lee, 2001). De fato, a memória de trabalho parece ser essencial à aprendizagem, nomeadamente de aptidões de leitura e de compreensão (de Jong, 1998; Gathercole et al., 2006; Oakhill, Yuill, \& Garnham, 2011; Pimperton \& Nation, 2010), bem como de aptidões matemáticas (Bull et al., 1999; Swanson \& Sachse-Lee, 2001), aptidões basilares em qualquer sistema educativo. Os deficit executivos, em particular os associados à atenção, inibição e memória de trabalho parecem estar relacionados não só com dificuldades de aprendizagem, como também com problemas comportamentais e emocionais (de Jong, 1998; Vuontela et al., 2013). Essas conclusões têm sido reiteradas, principalmente em populações clínicas como autistas (Russell, Jarrold, \& Henry, 1996), crianças hiperativas (Pliszka, Liotti, \& Woldorff, 2000) ou crianças com dificuldades de aprendizagem (Swanson \& Sachse-Lee, 2001).

Gathercole et al. (2006) conduziram um estudo que teve como principal objetivo investigar em que medida os deficit da memória de trabalho contribuem para as dificuldades apresentadas pelas crianças com perturbações de leitura. A amostra do estudo foi constituída por 46 crianças, com média de idades de 9 anos. Os participantes foram submetidos à aplicação de vários instrumentos, entre eles: Wechsler Objective Language Dimensions (que avalia a compreensão auditiva e a expressão oral), Wechsler Objective Numerical Dimensions (que avalia aptidões matemáticas), Wechsler Intelligence Scale for Children (que avalia o quociente de 
inteligência), Working Memory Test Battery for Children (que avalia a memória de trabalho em crianças), bem como a Phonological Assessment Battery (que avalia aspectos fonológicos). Os resultados sugeriram que os deficit da memória de trabalho representam uma restrição evidente na aquisição de conhecimentos de leitura e de matemática. Esses autores sugeriram ainda que as crianças com as aptidões de memória de trabalho pouco desenvolvidas têm possivelmente dificuldades no cumprimento de rotinas e de atividades estruturadas da sala de aula, i.e., têm influência não só ao nível da aprendizagem, mas também ao nível comportamental.

Começamos este artigo por falar sobre a atenção e é sobre esse assunto que voltamos a falar a seguir. Para se aprender, é necessário, em primeira instância, estar atento. O processamento de todas as informações que recebemos é manifestamente impossível, portanto, a cada momento, selecionamos as informações mais relevantes para uma dada tarefa e ignoramos as irrelevantes. Se pensarmos no nosso dia-a-dia, facilmente percebemos que recebemos constantemente uma grande variedade e quantidade de estímulos, quer externos, como estímulos visuais ou auditivos, quer internos, como, por exemplo, pensamentos automáticos negativos (Stevens \& Bavelier, 2012; Styles, 2005). A nossa capacidade de selecionarmos os estímulos relevantes e ignorarmos os distrativos não é sempre igual ao longo da nossa vida. As crianças constituem um grupo etário com maior vulnerabilidade para capturar informação irrelevante, o que as torna mais susceptíveis à distração, quando comparadas com os adultos. Essa susceptibilidade justifica-se pelo fato do seu sistema cognitivo, nomeadamente o controle top-down, se encontrar ainda em desenvolvimento (Craik \& Bialystok, 2006; Gaspelin, Margett-Jordan, \& Ruthruff, 2015).

São vários os estudos que referem que a atenção seletiva é essencial para o sucesso da aprendizagem (e.g., Garner, 2009; Mammarella \& Cornoldi, 2005). Vejamos em particular o estudo de Bosse e Valdois (2009). Esses autores realizaram um estudo transversal incluindo 417 crianças de vários estratos socioeconómicos e com média de idades a variar entre os 6,83 e os 10,92 anos. O principal objetivo dessa investigação foi estudar o papel da atenção visual na aprendizagem das aptidões de leitura. A bateria de testes utilizada incluiu tarefas de leitura, consciência fonológica e tarefas de atenção visual. Os resultados mostraram que a atenção visual pode explicar o progresso na aprendizagem da leitura, independentemente da influência da consciência fonológica. A atenção visual parece ter também um papel crucial a longo prazo na aquisição de conhecimentos específicos ortográficos. Ainda não se sabe contudo se os deficit atencionais que baseiam algumas dificuldades de aprendizagem ocorrem na modulação atencional ou ainda nos processos perceptivos anteriores (Stevens \& Bavelier, 2012).

Conforme referido anteriormente, a atenção refere-se à seleção da informação ambiental, que é necessária para uma dada tarefa em mãos. Segundo um estudo recente de Gaspelin et al. (2015), é necessária uma melhor compreensão dos processos de captação atencional nas crianças, o que permitirá desenhar ambientes educacionais mais ajustados. Contudo, os vários estudos que foram aqui mencionados, bem como a sua esmagadora maioria, ignoram o potencial efeito do ambiente circundante no desempenho das crianças, quer seja ao nível da aprendizagem, quer seja ao nível dos processos cognitivos anteriores que lhe estão subjacentes. Dito de uma outra forma, os processos de atenção e de memória são essenciais para uma boa aprendizagem, fato que está muito bem descrito na literatura, contudo a maioria dos estudos apresenta pouca validade ecológica. Poderemos assim refletir sobre o seguinte: se o processo de aprendizagem se refere à aquisição de informação externa e à sua conjugação com informações internas, será que algumas dificuldades de aprendizagem podem ser explicadas logo na interação indivíduo-ambiente? Mais importante do que respondermos a essa questão, é explorarmos um pouco o estado da literatura no que toca ao efeito do ambiente visual circundante na aprendizagem, assunto que é abordado na seção seguinte.

\section{O Ambiente Circundante Influencia os Nossos Processos Cognitivos?}

A influência das caraterísticas do ambiente circundante nos processos cognitivos não parece ser suficientemente explorada, embora se encontrem referências variadas à necessidade e importância de se levar em conta o ambiente nos contextos educacionais (e.g., Barrett et al., 2015; Cheryan, Ziegler, Plaut, \& Meltzoff, 2014). Algumas investigações têm concluído que vários aspectos ambientais influenciam a memorização da informação e, por conseguinte, a aprendizagem, nomeadamente o design do espaço, o número de pessoas nele presente, a sua cor e o barulho que se faz sentir (Ahrentzen \& Evans, 1984; Barrett et al., 2015; Cheryan et al., 2014; Stone, 2001). Também o uso de luz natural nas salas de aula, bem como a presença de ventilação natural são fatores que se associam a uma maior concentração dos alunos, isto é, o maior contato com elementos naturais parece ser um benefício para o desempenho escolar (Barrett et al., 2015). Efetivamente, são diversas as referências à importância da relação indivíduo-ambiente (Gifford, 2007), nomeadamente em contexto escolar (Gaspelin et al., 2015), embora a investigação pareça ainda escassa, especialmente no campo da Psicologia Cognitiva. Segundo Barrett et al. (2015), o desempenho acadêmico dos alunos é influenciado quer por fatores não ambientais, quer por fatores ambientais. Dos últimos, os autores destacam a naturalidade, a individualização e o nível de estimulação do espaço. A naturalidade refere-se aos aspectos que foram apontados anteriormente, i.e., o contato com elementos da natureza, a luz natural ou a temperatura são fatores a levar em conta nas escolas, porque exercem influência no bemestar e, por conseguinte, no processo de aprendizagem das crianças (Barrett et al., 2015). A individualização diz respeito a aspectos como a área e forma da sala de aula, a qualidade das mesas e cadeiras ou a existência/não existência de zonas de estudo apropriadas. Já a estimulação ambiental refere-se, entre outros, à diversidade visual que é apresentada na sala, nomeadamente objetos, posters, cores da parede ou número de quadros, apenas para dar alguns exemplos (Barrett et al., 2015; Cheryan et al., 2014). Partindo desse último parâmetro, 
há evidências de que elementos visuais presentes em uma sala podem facilitar o desempenho dos indivíduos (Latu, Mast, Lammers, \& Bombari, 2013) ou, por outro lado, prejudicá-lo (Fisher et al., 2014). Foquemo-nos nos estudos de Fisher e colaboradores $(2011,2014)$; esses investigadores concluíram que salas de aula com elevada carga visual, como posters, fotografias, quadros, entre outros elementos, parecem influenciar negativamente o desempenho das crianças nas suas tarefas escolares. Constatou-se que o ambiente visual da sala de aula pode ser o principal responsável pela alocação da atenção das crianças durante as aulas. Esse estudo consistiu em um design intra-sujeitos e resumiu-se ao seguinte: (a) foram criados dois ambientes de sala de aula, um com elevado número de elementos visuais distrativos (posters, fotografias, mapas e outros elementos visuais) e outro isento desses elementos distrativos; (b) formaram-se dois grupos de crianças, que passaram pelas duas condições ambientais de forma alternada; (c) em cada ambiente, foram ministradas aulas (cerca de 5 a 7 minutos) aos participantes e feitas algumas perguntas no final sobre o conteúdo das mesmas. Os resultados foram claros: (a) as crianças estiveram mais tempo desatentas no ambiente de elevada carga distrativa; (b) as crianças tiveram melhores desempenhos de aprendizagem no ambiente com poucos elementos distrativos. Importa referir que se designam por elementos distrativos todos os estímulos que, estando presentes em uma dada tarefa, não são necessários para o sucesso da mesma, devendo ser ignorados (Cassuto, Ben-Simon, \& Berger, 2013; Godwin \& Fisher, 2011; Rodrigues \& Pandeirada, 2015). Também Cassuto et al. (2013) chamaram à atenção para a necessidade de se levar em conta a validade ecológica das tarefas cognitivas utlizadas nas investigações sobre a distratibilidade. Segundo esses autores, que estudaram o potencial dos distratores visuais e auditivos no diagnóstico de hiperatividade, as crianças, quando estão em aula a realizar um trabalho, devem ignorar outros estímulos, nomeadamente o barulho que vem da sala ao lado, portanto, é com esse tipo de estímulos que as investigações devem ser realizadas. Todavia, a investigação de Cassuto et al. (2013), embora utilizasse estímulos semelhantes aos encontrados pelas crianças no seu dia-a-dia (nomeadamente visuais), apresentou-os em monitores, o que se diferencia em grande medida das investigações de Fisher e seus colaboradores (2014).

Como referimos anteriormente, os processos atencionais e mnemônicos são fundamentais para a aprendizagem dos alunos. É em contexto de sala de aula, que pode ser favorável ou desfavoravelmente estimulante, que os alunos se encontram diariamente para aprender. Aproximar os procedimentos experimentais aos contextos reais de sala de aula parece pois fundamental para se perceber melhor a sua influência na aprendizagem. Antes, parece ainda importante percebermos de que modo o ambiente circundante influencia as nossas funções cognitivas básicas, que estão subjacentes às diversas tarefas do cotidiano, entre as quais a aprendizagem. Parece-nos, assim, importante a realização de investigações tendo por base os estudos de Rodrigues e Pandeirada (2015). Esse estudo, realizado com uma amostra de idosos, assemelha-se em grande medida aos estudos de
Fisher et al. (2014) pela manipulação visual do ambiente externo. $\mathrm{O}$ estudo foi realizado com 40 idosos portugueses, que realizaram duas sessões experimentais com intervalo de 14 a 21 dias entre elas. Uma das sessões foi realizada em ambiente a que os autores designaram de distrativo (sala com imagens e posters coloridos) e a outra, em ambiente não distrativo (sala isenta dos elementos visuais referidos). Em cada sessão, cada participante, de forma individual, realizou duas tarefas atencionais do foro visual e três tarefas de memória de trabalho da Escala de Inteligência de Wechsler para Adultos - III (WAIS-III; Wechsler, 2008). A ordem de realização das tarefas, assim como a ordem de manipulação do ambiente foi contrabalanceada entre os participantes. Os resultados indicaram um melhor desempenho dos idosos no ambiente não distrativo, em comparação com o desempenho verificado no ambiente distrativo, resultado mais evidente nas tarefas atencionais realizadas. Importa referir que as tarefas atencionais administradas foram de cariz visual, enquanto que as tarefas de memória foram de cariz verbal. Esse estudo veio alertar para a relação indivíduo-ambiente, em particular a influência que o ambiente visual circundante pode ter em tarefas cognitivas visuais.

Estudos semelhantes aos de Rodrigues e Pandeirada (2015) deverão ser projetados em populações escolares, uma vez que englobam duas dimensões importantes do processo de aprendizagem. A primeira é que nos dá a possibilidade de estudarmos processos cognitivos básicos avaliados por tarefas específicas que estão na base da aprendizagem. Em segundo, porque esse procedimento nos permite a inserção dessas tarefas em contextos ecologicamente mais válidos. Dito de outra forma, com esse procedimento de investigação, aproximam-se as validades interna e externa em um mesmo estudo: validade interna ao utilizarem-se tarefas cognitivas empiricamente validadas e controladas; validade externa, porque se inserem as tarefas cognitivas em um contexto visual circundante mais próximo do encontrado em salas de aula. De fato, Davids (1988) defendeu que a investigação deve maximizar a validade externa, não pondo em causa a sua validade interna. $\mathrm{O}$ mesmo autor propõe, nesse sentido, três critérios que devem estar na base da validade ecológica da investigação científica: realismo, união e análise eclética. $\mathrm{O}$ primeiro defende que o ambiente experimental deve aproximar-se o máximo possível do contexto real em que o comportamento estudado habitualmente se insere. O segundo refere-se à necessidade de convergência entre validade interna e o processo de investigação no terreno. Já a análise eclética diz respeito às técnicas que se devem utilizar para garantir que pelo menos um aspecto do comportamento estudado é analisado em contexto real.

Tendo em conta as investigações que se referiram, bem como a necessidade vincada na literatura de se apostarem em investigações ecologicamente mais válidas do que até aqui se têm realizado, defendemos um procedimento de investigação conforme a ilustração esquemática seguinte (Figura 1), nomeadamente em populações escolares. 


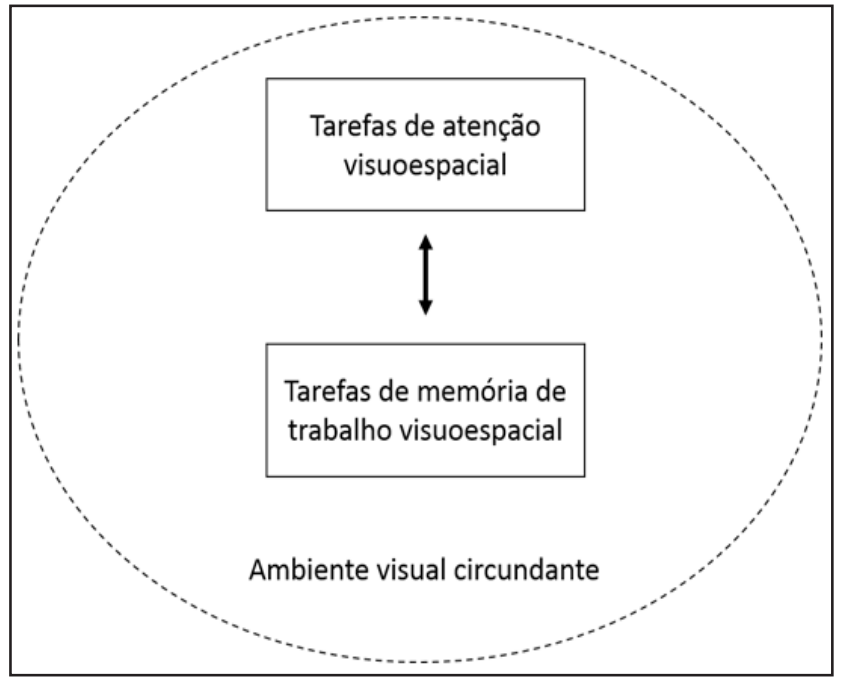

Figura 1. Procedimento de investigação em que se usam tarefas cognitivas visuoespaciais, em um contexto visual circundante manipulável. Nesta ilustração, é evidente a estreita relação entre os processos de atenção e de memória de trabalho visuoespaciais, tal como descrito na literatura; Contudo é acrescentada a manipulação visual do ambiente circundante (cf. Fisher et al., 2014; Rodrigues \& Pandeirada, 2015).

\section{Considerações Finais}

O desempenho acadêmico é determinado por uma variedade de fatores, nomeadamente as oportunidades educacionais, o status socioeconômico, as aptidões sociais, os traços de personalidade e as capacidades cognitivas (e.g., Brooks-Gunn \& Duncan, 1997; Engel de Abreu, Puglisi, Cruz-Santos, Befi-Lopes, \& Martin, 2014). As aptidões acadêmicas das crianças não parecem ser determinadas apenas pelas oportunidades de aprendizagem, mas são sobretudo influenciadas pelos processos cognitivos básicos individuais. Esse fato justifica em grande medida que os vários alunos de uma mesma sala de aula, cujo ambiente de aprendizagem é semelhante, tenham habitualmente desempenhos escolares diferentes. Dito de outra forma, parece vital que os fatores ambientais e as capacidades individuais sejam considerados em conjunto, uma vez que estão interligados no processo de aprendizagem (Gathercole, Pickering, Knight, \& Stegmann, 2004). Como podemos verificar com a nossa revisão de literatura, a atenção seletiva, a memória de trabalho e a inibição têm um papel fundamental no processo de aprendizagem e isso já está bem descrito na literatura (e.g., St Clair-Thompson \& Gathercole, 2006; Vuontela et al., 2013). Os indivíduos com maior capacidade de memória de trabalho parecem ser mais capazes de suprimir itens irrelevantes e codificar apenas os itens relevantes apresentados, ao invés dos indivíduos com pior capacidade de memória de trabalho, que codificam mais itens na memória, incluindo itens irrelevantes. O controle da atenção seletiva e da inibição é importante para regular o acesso à memória de trabalho e, portanto, para otimizar a capacidade mnemônica (Vogel, McCollough, \& Machizawa, 2005). Estudos semelhantes ao de Fisher et al. (2014) são necessários para se averiguar o efeito dos ambientes visualmente estimulantes na aprendizagem. Se efetivamente as conclusões desse último estudo se reiterarem, será necessário perceber o que acontece ao nível cognitivo básico, isto é, qual o papel dos elementos ambientais circundantes em tarefas cognitivas básicas, tais como atenção e memória. Neste campo, que seja do nosso conhecimento, apenas um estudo publicado (com idosos) aborda o papel de elementos visuais do ambiente circundante em tarefas cognitivas de atenção e memória de trabalho (Rodrigues \& Pandeirada, 2015). Nesse estudo, os investigadores verificaram que, sobretudo em tarefas atencionais do foro visual, os idosos apresentaram piores resultados quando as realizaram em ambiente distrativo (ambiente circundante visualmente estimulante), em comparação com o ambiente não distrativo. Sendo a atenção um processo cognitivo básico e subjacente à aprendizagem, o que acontecerá com as crianças? Do ponto de vista desenvolvimental, tal como abordamos, a capacidade atencional nas crianças encontrase em aperfeiçoamento, o que faz com que ignorem menos informação irrelevante quando comparadas com os adultos cujas capacidades cognitivas se encontram no pico do seu desenvolvimento. Os idosos também apresentam dificuldades atencionais, mas fruto do normal declínio funcional que o envelhecimento acarreta (Craik \& Bialystok, 2006). Pressupondo que o ambiente tem um papel no desempenho acadêmico das crianças, qual o seu modelo explicativo? Poderão as mesmas linhas explicativas dos estudos referidos neste artigo (que não tiveram em conta a dimensão ecológica) serem aplicadas a estudos ecológicos? Pressupomos que, à semelhança dos idosos (Rodrigues \& Pandeirada, 2015), as crianças apresentem também dificuldades em ignorar estímulos irrelevantes que se encontram no ambiente (situação que ocorre ao nível da atenção). Uma vez que a atenção está estreitamente relacionada com os processos de memória, designadamente com a memória de trabalho, e, por conseguinte, com a aprendizagem (Garner, 2009; Styles, 2005), serão cruciais mais pesquisas que considerem a validade ecológica, o que explicará com maior exatidão o que ocorre nas salas de aula. Se for verificado em crianças o que Rodrigues e Pandeirada (2015) verificaram nas tarefas atencionais visuais (em idosos), poderemos justificar os resultados de Fisher et al. (2014). Dito de outra forma, parece que os elementos visuais ambientais têm impacto na aprendizagem das crianças, mas que modelo explicativo poderemos apontar para essa influência? Poderemos supor que elementos presentes no contexto de sala de aula influenciam em primeira instância os processos cognitivos básicos subjacentes à aprendizagem, o que se reflete em menor aprendizagem dos conteúdos das aulas. Assim, o estudo de Rodrigues e Pandeirada (2015), ao abordar o efeito ambiental externo em processos cognitivos básicos, responde a questões ainda mais basilares do que os estudos de Fisher et al. (2014), sendo ambas as linhas de investigação muito importantes e complementares.

Concluindo este trabalho de síntese, é inegável que a atenção seletiva, a inibição e a memória de trabalho sejam processos cognitivos fundamentais no processo de aprendizagem. Investigações sobre a primeira fase da aprendizagem, i.e., a aquisição informacional, que requer recursos atencionais intactos, são bem-vindas, uma vez que o conhecimento dos processos que ocorrem na interação indivíduo-ambiente contribuirá certamente para a melhoria dos sistemas de ensino e para a constituição de programas de intervenção mais direcionados e eficazes. Propõe-se, assim, 
um paradigma de investigação que alie tarefas cognitivas empiricamente validadas, inseridas em um ambiente externo manipulável, o que permitirá à comunidade científica conclusões com maior validade ecológica e, por isso, mais aplicadas aos contextos reais.

\section{Referências}

Ahrentzen, S., \& Evans, G. W. (1984). Distraction, privacy, and classroom design. Environment and Behavior, 16, 437-454. doi:10.1177/0013916584164002

Amso, D., \& Scerif, G. (2015). The attentive brain: Insights from developmental cognitive neuroscience. Nature Reviews Neuroscience, 16, 606-619. doi:10.1038/nrn4025

Aronen, E., Vuontela, V., Steenari, M.-R., Salmi, J., \& Carlson, S. (2005). Working memory, psychiatric symptoms, and academic performance at school. Neurobiology of Learning and Memory, 83, 33-42. doi:10.1016/j.nlm.2004.06.010

Arrington, C. N., Kulesz, P. A., Francis, D. J., Fletcher, J. M., \& Barnes, M. A. (2014). The contribution of attentional control and working memory to reading comprehension and decoding. Scientific Studies of Reading, 18, 325-346. doi:10.1080/1088 8438.2014.902461

Baddeley, A. (1992). Working memory. Science, 255(5044), 556559. doi: 10.1126/science. 1736359

Baddeley, A. (2010). Working memory. Current Biology, 20, 136140. doi:10.1016/j.cub.2009.12.014

Baddeley, A. (2012). Working memory: Theories, models, and controversies. Annual Review of Psychology, 63, 1-29. doi:10.1146/annurev-psych-120710-100422

Barkley, R. A. (1997). Behavioral inhibition, sustained attention, and executive functions: Constructing a unifying theory of ADHD. Psychological Bulletin, 121, 65-94. doi:10.1037/00332909.121.1.65

Barkley, R.A. (2001). The executive functions and self-regulation: An evolutionary neuropsychological perspective. Neuropsychology Review, 11, 1-29. doi:10.1023/A:1009085417776

Barrett, P., Davies, F., Zhang, Y., \& Barrett, L. (2015). The impact of classroom design on pupils' learning: Final results of a holistic, multi-level analysis. Building and Environment, 89, 118-133. doi:10.1016/j.buildenv.2015.02.013

Blair, C., \& Razza, R. P. (2007). Relating effortful control, executive function, and false belief understanding to emerging math and literacy ability in kindergarten. Child Development, 78, 647663. doi:10.1111/j.1467-8624.2007.01019.x

Boekaerts, M. (1996). Self-regulated learning at the junction of cognition and motivation. European Psychologist, 1, 100-112. doi:10.1027/1016-9040.1.2.100

Booth, J. R., Burman, D. D., Meyer, J. R., Lei, Z., Trommer, B. L., Davenport, N. D., ... Mesulam, M. (2003). Neural development of selective attention and response inhibition. NeuroImage, 20, 737-751. doi:10.1016/S1053-8119(03)00404-X

Borella, E., Carretti, B., \& De Beni, R. (2008). Working memory and inhibition across the adult life-span. Acta Psychologica, 128, 33-44. doi: 10.1016/j.actpsy.2007.09.008

Bosse, M. L., \& Valdois, S. (2009). Influence of the visual attention span on child reading performance: A cross-sectional study. Journal of Research in Reading, 32, 230-253. doi:10.1111/ j.1467-9817.2008.01387.x
Brooks-Gunn, J., \& Duncan, G. J. (1997). The effects of poverty on children. The Future of Children, 7, 55-71. doi: $10.2307 / 1602387$

Bull, R., Johnston, R. S., \& Roy, J. A. (1999). Exploring the roles of the visual-spatial sketch pad and central executive in children's arithmetical skills: Views from cognition and developmental neuropsychology. Developmental Neuropsychology, 15, 421442. doi: 10.1080/87565649909540759

Carlson, S., Martinkauppi, S., Rämä, P., Salli, E., Korvenoja, A., \& Aronen, H. J. (1998). Distribution of cortical activation during visuospatial n-back tasks as revealed by functional magnetic resonance imaging. Cerebral Cortex, 8, 743-752. doi:10.1093/ cercor/8.8.743

Cassuto, H., Ben-Simon, A., \& Berger, I. (2013). Using environmental distractors in the diagnosis of ADHD. Frontiers in Human Neuroscience, 7, 46-55. doi:10.3389/ fnhum.2013.00805

Cheryan, S., Ziegler, S. A., Plaut, V. C., \& Meltzoff, A. N. (2014). Designing classrooms to maximize student achievement. Policy Insights from the Behavioral and Brain Sciences, 1, 4-12. doi:10.1177/2372732214548677

Craik, F. I. M., \& Bialystok, E. (2006). Cognition through the lifespan: mechanisms of change. Trends in Cognitive Sciences, 10, 131-138. doi:10.1016/j.tics.2006.01.007

Davids, K. (1988). Ecological validity in understanding sport performance: Some problems of definition. Quest, 40, 126-136. doi:10.1080/00336297.1988.10483894

de Fockert, J. W., Rees, G., Frith, C. D., \& Lavie, N. (2001). The role of working memory in visual selective attention. Science, 291(5509), 1803-1806. doi:10.1126/science.1056496

de Jong, P. F. (1998). Working memory deficits of reading disabled children. Journal of Experimental Child Psychology, 70, 75-96. doi:10.1006/jecp.1998.2451

Diamond, A., Barnett, W. S., Thomas, J., \& Munro, S. (2007). Preschool program improves cognitive control. Science, 318(5855), 1387-1388. doi:10.1126/science. 1151148

Engel de Abreu, P. M. J., Puglisi, M. L., Cruz-Santos, A., BefiLopes, D. M., \& Martin, R. (2014). Effects of impoverished environmental conditions on working memory performance. Memory, 22, 323-331. doi:10.1080/09658211.2013.781186

Feldman, D. E. (2009). Synaptic mechanisms for plasticity in neocortex. Annual Review of Neuroscience, 32, 33-55. doi:10.1146/annurev.neuro.051508.135516

Fisher, A. V., Godwin, K. E., \& Seltman, H. (2014). Visual environment, attention allocation, and learning in young children: When too much of a good thing may be bad. Psychological Science, 25, 1362-1370. doi:10.1177/0956797614533801

Friso-van den Bos, I., van der Ven, S. H. G., Kroesbergen, E. H., \& van Luit, J. E. H. (2013). Working memory and mathematics in primary school children: A meta-analysis. Educational Research Review, 10, 29-44. doi:10.1016/j.edurev.2013.05.003

Fuster, J. M. (2002). Frontal lobe and cognitive development. Journal of Neurocytology, 31, 373-385. doi:10.1023/A:1024190429920

Gallistel, C. R. (2000). The replacement of general-purpose learning models with adaptively specialized learning modules. In M. S. Gazzaniga (Ed.), The cognitive neurosciences (pp. 1179-1191). Cambridge, MA: MIT Press.

Garner, J. K. (2009). Conceptualizing the relations between executive functions and self-regulated learning. The Journal of Psychology, 143, 405-426. doi:10.3200/JRLP.143.4.405-426 
Gaspelin, N., Margett-Jordan, T., \& Ruthruff, E. (2015). Susceptible to distraction: Children lack top-down control over spatial attention capture. Psychonomic Bulletin \& Review, 22, 461468. doi:10.3758/s13423-014-0708-0

Gathercole, S. E., Alloway, T. P., Kirkwood, H. J., Elliott, J. G., Holmes, J., \& Hilton, K. A. (2008). Attentional and executive function behaviours in children with poor working memory. Learning and Individual Differences, 18, 214-223. doi:10.1016/j.lindif.2007.10.003

Gathercole, S. E., Alloway, T. P., Willis, C., \& Adams, A.-M. (2006). Working memory in children with reading disabilities. Journal of Experimental Child Psychology, 93, 265-281. doi:10.1016/j. jecp.2005.08.003

Gathercole, S. E., Pickering, S. J., Knight, C., \& Stegmann, Z. (2004). Working memory skills and educational attainment: Evidence from national curriculum assessments at 7 and 14 years of age. Applied Cognitive Psychology, 18, 1-16. doi:10.1002/acp.934

Gazzaley, A., \& Nobre, A. C. (2012). Top-down modulation: Bridging selective attention and working memory. Trends in Cognitive Sciences, 16, 129-135. doi:10.1016/j.tics.2011.11.014

Gifford, R. (2007). Environmental psychology and sustainable development: Expansion, maturation, and challenges. Journal of Social Issues, 63, 199-212. doi:10.1111/j.15404560.2007.00503.x

Gleitman, H., Fridlund, A. J., \& Reisberg, D. (2007). Psicologia. Lisboa: Fundação Calouste Gulbenkian.

Godwin, K. E., \& Fisher, A. V. (2011). Allocation of attention in classroom environments: Consequences for learning. Paper presented at The Annual Meeting of the Cognitive Science Society, Boston, Massachusetts.

Jurado, M., \& Rosselli, M. (2007). The elusive nature of executive functions: A review of our current understanding. Neuropsychology Review, 17, 213-233. doi:10.1007/s11065007-9040-z

Katsuki, F., \& Constantinidis, C. (2012). Early involvement of prefrontal cortex in visual bottom up attention. Nature Neuroscience, 15, 1160-1166. doi:10.1038/nn.3164

Kirkham, N. Z., Cruess, L., \& Diamond, A. (2003). Helping children apply their knowledge to their behavior on a dimension-switching task. Developmental Science, 6, 449-467. doi:10.1111/1467-7687.00300

Lachman, R., Lachman, J. L., \& Butterfield, E. C. (2015). Cognitive psychology and information processing: An introduction. New York: Psychology Press.

Latu, I. M., Mast, M. S., Lammers, J., \& Bombari, D. (2013). Successful female leaders empower women's behavior in leadership tasks. Journal of Experimental Social Psychology, 49, 444-448. doi:10.1016/j.jesp.2013.01.003

Mammarella, I. C., \& Cornoldi, C. (2005). Difficulties in the control of irrelevant visuospatial information in children with visuospatial learning disabilities. Acta Psychologica, 118, 211-228. doi:10.1016/j.actpsy.2004.08.004

Mammarella, I. C., Cornoldi, C., Pazzaglia, F., Toso, C., Grimoldi, M., \& Vio, C. (2006). Evidence for a double dissociation between spatial-simultaneous and spatial-sequential working memory in visuospatial (nonverbal) learning disabled children. Brain and Cognition, 62, 58-67. doi:10.1016/j. bandc.2006.03.007
Miller, E. K., \& Cohen, J. D. (2001). An integrative theory of prefrontal cortex function. Annual Review of Neuroscience, 24, 167-202. doi:10.1146/annurev.neuro.24.1.167

Nelson, D. B., \& Low, G. R. (2003). Emotional intelligence: Achieving academic and career excellence. Upper Saddle River, New Jersey: Prentice Hall.

Oakhill, J., Yuill, N., \& Garnham, A. (2011). The differential relations between verbal, numerical and spatial working memory abilities and children's reading comprehension. International Electronic Journal of Elementary Education, 4, 83-106.

Petersen, S. E., \& Posner, M. I. (2012). The attention system of the human brain: 20 years after. Annual Review of Neuroscience, 35, 73-89. doi:10.1146/annurev-neuro-062111-150525

Pimperton, H., \& Nation, K. (2010). Suppressing irrelevant information from working memory: Evidence for domainspecific deficits in poor comprehenders. Journal of Memory and Language, 62, 380-391. doi:10.1016/j.jml.2010.02.005

Pinto, A. C. (2001). Memória, cognição e educação: Implicações mútuas. In B. Detry \& F. Simas (Eds.), Educação, cognição e desenvolvimento: Textos de psicologia educacional para a formação de professores (pp. 17-54). Lisboa: Edinova.

Pliszka, S. R., Liotti, M., \& Woldorff, M. G. (2000). Inhibitory control in children with attention-deficit/hyperactivity disorder: Event-related potentials identify the processing component and timing of an impaired right-frontal response-inhibition mechanism. Biological Psychiatry, 48, 238-246. doi:10.1016/ S0006-3223(00)00890-8

Posner, M. I. (2011). Cognitive neuroscience of attention. New York: Guilford Press.

Radvansky, G. (2011). Human memory (2nd ed.). Boston: Pearson Education.

Rodrigues, P. F. S., \& Pandeirada, J. N. S. (2015). Attention and working memory in elderly: The influence of a distracting environment. Cognitive Processing, 16, 97-109. doi:10.1007/ s10339-014-0628-y

Russell, J., Jarrold, C., \& Henry, L. (1996). Working memory in children with autism and with moderate learning difficulties. Journal of Child Psychology and Psychiatry, 37, 673-686. doi:10.1111/j.1469-7610.1996.tb01459.x

Rutman, A. M., Clapp, W. C., Chadick, J. Z., \& Gazzaley, A. (2010). Early top-down control of visual processing predicts working memory performance. Journal of Cognitive Neuroscience, 22, 1224-1234. doi:10.1162/jocn.2009.21257

St Clair-Thompson, H. L., \& Gathercole, S. E. (2006). Executive functions and achievements in school: Shifting, updating, inhibition, and working memory. The Quarterly Journal of Experimental Psychology, 59, 745-759. doi:10.1080/17470210500162854

Stevens, C., \& Bavelier, D. (2012). The role of selective attention on academic foundations: A cognitive neuroscience perspective. Developmental Cognitive Neuroscience, 2(Supplement 1), S30-S48. doi:10.1016/j.den.2011.11.001

Stone, N. J. (2001). Designing effective study environments. Journal of Environmental Psychology, 21, 179-190. doi:10.1006/ jevp. 2000.0193

Styles, E. A. (2005). Attention, perception and memory. An integrated introdution (1st ed.). New York: Psychology Press. 
Swanson, H. L., \& Sachse-Lee, C. (2001). Mathematical problem solving and working memory in children with learning disabilities: Both executive and phonological processes are important. Journal of Experimental Child Psychology, 79, 294-321. doi:10.1006/jecp.2000.2587

Tavares, J., Pereira, A. S., Gomes, A. A., Monteiro, S. M., \& Gomes, A. (2007). Manual de Psicologia do Desenvolvimento e Aprendizagem ( $1^{\mathrm{a}}$ ed.). Porto: Porto Editora.

Terry, K. M. (1995). Selective attention and the inhibitory control of cognition. In Frank N. Dempster \& Charles J. Brainerd (Eds.), Interference and inhibition in cognition (pp. 207-261). San Diego: Academic Press, Inc.

Vogel, E. K., McCollough, A. W., \& Machizawa, M. G. (2005). Neural measures reveal individual differences in controlling access to working memory. Nature, 438, 500-503. doi:10.1038/ nature 04171
Vuontela, V., Carlson, S., Troberg, A. M., Fontell, T., Simola, P., Saarinen, S., \& Aronen, E. T. (2013). Working memory, attention, inhibition, and their relation to adaptive functioning and behavioral/emotional symptoms in school-aged children. Child Psychiatry \& Human Development, 44, 105-122. doi:10.1007/s10578-012-0313-2

Wechsler, D. (2008). WAIS-III: Escala de inteligência de Wechsler para adultos ( $3^{\mathrm{a}}$ ed.). Lisboa: Cegoc.

Zanto, T. P., Hennigan, K., Östberg, M., Clapp, W. C., \& Gazzaley, A. (2010). Predictive knowledge of stimulus relevance does not influence top-down suppression of irrelevant information in older adults. Cortex, 46, 564-574. doi:10.1016/j. cortex.2009.08.003

Zelazo, P. D., Craik, F. I. M., \& Booth, L. (2004). Executive function across the life span. Acta Psychologica, 115(2-3), 167-183. doi:10.1016/j.actpsy.2003.12.005

Recebido em 15.07.2015

Primeira decisão editorial em 29.08.2016

Versão final em 10.10.2016

Aceito em 26.10.2016 